\title{
Geometric Scales and Force Fields
}

\author{
Thekkumkattil Madathil Vasudevan \\ Independent Researcher, Cochin, India \\ Email: tmvnambiar08@gmail.com
}

Received 26 November 2013; revised 25 December 2013; accepted 3 January 2014

Copyright (C) 2014 by author and Scientific Research Publishing Inc.

This work is licensed under the Creative Commons Attribution International License (CC BY).

http://creativecommons.org/licenses/by/4.0/

(c) (1) Open Access

\section{Abstract}

This is an attempt to view the concept of quantization of Geometry in a very different way from the prevailing views on the subject. It is postulated that the quantum levels of geometry form a geometric progression (like $\left.a, a x, a x^{2}, a x^{3}, a x^{4}, \cdots, a x^{n}\right)$ where the scale factor " $a$ " stands for $l_{\mathrm{P}} / 2\left(\mathrm{l}_{\mathrm{P}}=\right.$ $1.616199 \times 10^{-35} \mathrm{~m}$ is the Planck's length) and the common ratio " $x$ " stands for $\mathrm{e}^{(\sqcap \sqrt{2}) / 3}$. Based on observational facts, it is further attempted to establish that the Geometric Quantum levels could be grouped into different scales, namely, pre-atomic scale, atomic scale, cosmic scale, super-cosmic scale, etc., with the accompanying force fields. It is further postulated that detection of any super cosmic structure with a length or diameter of the order of magnitude of 20 Billion Light Years would mean that a super-cosmic scale is present beyond the observable Universe. This paper just describes a proposed theoretical framework which could ultimately explain all the observable phenomena, in the Universe, without venturing into a detailed mathematical study to support the theory.

\section{Keywords}

Geometric Scales; Force-Fields; Super-Cosmic; Pre-Atomic

\section{Introduction}

There have been so many different theories, to explain the observable phenomena in the Universe, but none of these could be called the ultimate theory, so far, due to limitations in scope as well as lack of sufficient supporting experimental evidences. This is also an attempt to look at the things from a totally different perspective, to formulate a model for explaining all the observable physical phenomena in the Universe. Related research was done by the present author some time ago and a paper was published in the Journal Philica in the year 2006 [1]. The equation-33 mentioned in this paper forms the basis of the discussions in the present theory.

The basic assumption of the present theory is that the three spatial dimensions of the most elementary volume of space-time start with the Planck's Length which is $\ell_{\mathcal{P}}=1.616199 \times 10^{-35} \mathrm{~m}$ [2]. In other words, if we consider 
a circle with diameter equal to $\ell_{\mathcal{p}}$ then that would be the smallest circle that could be physically drawn. In the same way if we consider that a sphere with $\ell_{p}$ as the diameter is representing the smallest unit of spatially bounded energy then using a formula, which is postulated in this theory, we are making an attempt to derive the spatial dimensions of all other structures in the Universe. The formula is given below and so is the diagrammatic representation (Figure 1) (not to scale).

By putting $n=32$ we get $R_{32}=3.0832351 \times 10^{-15} \mathrm{~m}$ which is close to the radius of an electron $\left(r_{e}=2.81794 \times\right.$ $10^{-15} \mathrm{~m}$ ) [3], the smallest known elementary particle other than electron neutrino. If we postulate that Atomic scale is starting with electron, then we can say that all the geometric quantum levels starting from $R_{0}$ and ending with $R_{31}$ belong to the pre-atomic scale. Going further ahead in the same way if we calculate $R_{n}$ by putting $n=$ 64 we get $R_{64}=1176.3822 \mathrm{~km}$ which is in the range of the radius of a planet. In place of 64 if we put $n=63$ we get $R_{63}=267.5317 \mathrm{~km} \mathrm{[4]}$ which is in the range of the radius of a dwarf-planet. If we postulate that the cosmic scale is starting with planets, then we can say that all the geometric quantum levels starting from $R_{32}$ and ending with $R_{63}$ belong to the atomic scale. Also we can consider a quasi-planet as a transitional object between the atomic \& cosmic scales. Continuing in the same fashion if we put $n=96$ then we get $R_{96}=47.4419332$ Billion Light Years which happens to be just above the estimated limits of the observable Universe (the current co-moving distance to the edge of the observable universe is estimated to be 46.6 billion light years [5]). If we postulate that the super-cosmic scale is starting with $R_{96}$, then we can say that all the geometric quantum levels starting from $R_{64}$ and ending with $R_{95}$ belong to the cosmic scale. Taking the example of quasi-planet we could expect the existence of a transitional object between the cosmic $\&$ the super-cosmic scales with a radius of $R_{95}$. On calculating the value of $R_{95}$, we get $R_{95}=10.7892$ Billion Light Years for the radius of this transitional object. In other words, detection of any cosmic hyper structure with spatial dimensions comparable to 21.5784 Billion Light Years $\left(2 \times R_{95}\right)$ will indirectly hint at the existence of a super-cosmic scale starting with a radius of $R_{96}=$ 47.4419332 Billion Light Years.

\section{Scales \& Force-Fields}

Now that we have postulated the grouping of the geometric quantum levels, starting from Planck's length, into different scales (we have restricted to only 4 different scales as anything beyond the $4^{\text {th }}$ scale would be unimaginable), we need to further discuss about the force fields accompanying the different geometric scales also. Please find below the proposed classification of the force fields based on the scales as discussed above.

\section{Pre-atomic}

1. Electro-magnetic

2. Week Force

\section{Atomic}

1. Electro-magnetic

2. Week Force

3. Strong Force (Nuclear)

4. Gravitational (basic)

\section{Cosmic}

1. Electro-magnetic

2. Week Force

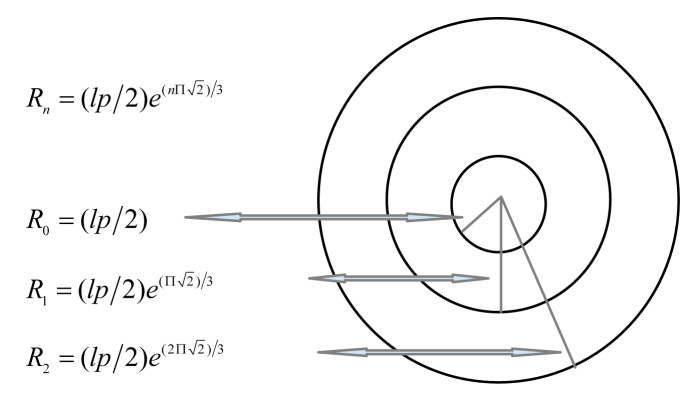

Figure 1. Dimensions of the structures in the universe. 


\section{Strong Force (Nuclear) \\ 4. Gravitational (basic) \\ 5. Strong force (cosmic) \\ 6. Gravitational (cosmic) \\ Super-cosmic}

1. Electro-magnetic

2. Week Force

3. Strong Force (Nuclear)

4. Gravitational (basic)

5. Strong force (cosmic)

6. Gravitational (cosmic)

7. Strong Force (super-cosmic)

8. Gravitational (super-cosmic)

Taking the case of pre-atomic scale first, the only force fields acting at such short ranges are Electromagnetic Force \& Weak Nuclear Force (with a range between $10^{-16} \mathrm{~m}$ and $10^{-18} \mathrm{~m}$ ). Coming to the atomic scale there are two more additional force fields, namely, Strong Nuclear Force and Gravitational Force. Since the range of Strong Nuclear Force is from $0.7 \times 10^{-15} \mathrm{~m}$ to $2.5 \times 10^{-15} \mathrm{~m}$, which is just below the starting value of the atomic scale $\left(R_{32}=3.0832351 \times 10^{-15} \mathrm{~m}\right)$, it could be considered as the manifestation of a transitional force. Coming to the cosmic scale, in addition to the other 4 force fields, there are two more force fields predicted. One of these force fields, which we call as Strong Force (Cosmic), will be similar to the Strong Nuclear Force Field, but will be acting in a range between $267 \mathrm{~km} \& 1176 \mathrm{~km}$. This strong force field could be the reason behind the formation of planets and other heavenly bodies and could also be responsible for the very high amounts of energy observed during the explosion of such objects. This force also, like the Strong Nuclear Force, could be considered as the manifestation of a transitional force. The sixth force field which is presently known as Dark Matter/Dark Energy (for example, brought in to explain the flattening of the Galaxy rotation curves, etc.) will be similar in nature to Gravitational Force Field and can be called Gravitational force (cosmic). Continuing the same line of argument for the super-cosmic scale, apart from the six force fields already described, there will be two more force fields, namely, Strong Force (super-cosmic) \& Gravitational (Super-cosmic) Force. The Strong Force (Super-cosmic) will also be a transitional force, like the two transitional forces already described, and will be active in a range between 10.7892 Billion Light Years and 47.4419332 Billion Light Years. The eighth force field, designated as Gravitational Force (Super-cosmic), will start manifesting beyond a distance of 47.4419332 Billion Light Years, which will be well beyond the observable Universe (as per the present estimates) and hence it is difficult to observe any direct evidence of its manifestation in the observable Universe.

\section{Formation of Planets \& Stars}

We attributed the reason behind formation of planets to the manifestation of a New Strong Force (Cosmic) and it is very likely that the anticyclonic vortexes observed in nature are also caused by this. Many of the stable anticyclonic vortexes observed are in the range of $1000 \mathrm{~km}$ and above in diameter which is in agreement with the predicted range of the Strong Cosmic Force. Once it is accepted that a strong force field comes into play, just like the strong nuclear force field, with the center of the vortex as the origin then it is pretty simple to understand how star or planet formation takes place out of the interstellar dust cloud surrounding the vortex. Star formation takes place first and later planets form from the surrounding interstellar dust cloud forming planetary systems. The quantity of the interstellar dust at any particular location as also the density of the dust cloud decide whether it is going to be a lone/ binary star, a planetary system or a lone planet. Vortexes are seen aplenty in the universe, be it on earth, Jupiter (for example, Great Red Spot) or even Sun (Sun spots) and it becomes very easy to explain all such phenomena if we accept the existence of a Strong force acting towards the center of the vortex.

\section{Accelerated Expansion of the Universe}

The accelerated expansion has been estimated to happen between 7.5 billion years to 10 billion years after the Big Bang. As there are no convincing methods of reconciling this observation, with the known effects of Gravitational Force, it came to be attributed to some mysterious force called Dark Energy. It may be recalled from our earlier discussions that the New Strong Force (Super-cosmic) will become active in a range between 10.7892 
Billion Light Years and 47.4419332 Billion Light Years. Since the effect of such a force will be to impart outward acceleration on matter the acceleration is expected to be felt at a distance of approximately 12.6 billion LY (provided 46.6 billion LY is taken as the outer limit of the observable Universe) and is expected to continue till a distance of approximately 29 billion LY. Beyond this distance the acceleration will stop and deceleration will start and continue till a distance of 47.4419332 billion LY. If there are any hints of the accelerated expansion slowing down beyond the distance of 29 billion LY, then it would be the direct evidence of the New Strong Force (Super-cosmic) as described.

\section{Conclusion}

The geometric progression of lengths starting with $\ell_{\mathcal{p}} / 2$, as described, has been derived from the equation of the logarithmic spiral represented by the formula, $R=a \mathrm{e}^{(b \theta)}$, where " $a$ " stands for $\left(\ell_{\mathcal{D}} / 2\right)$ and " $b$ " stands for $(\sqrt{2} / 3)$. In the theory, only the discrete values, namely, $\theta=n \pi$ (where $n=0,1,2,3, \cdots, 96$ ) have been used for deriving the lengths. The purpose of this paper is not to present a complete theory with the proper mathematical proofs, experimental data, etc., but, it is rather an attempt to look at the Grand design of the Universe from an entirely different perspective.

\section{References}

[1] Vasudevan, T. (2006) An Attempt on TOE-Part-I. Astro.philica.com. Article No. 21, 8.

[2] NIST (National Institute of Standards and Technology, US Department of Commerce), Planck Length. NIST's Published CODATA Constants.

[3] NIST (National Institute of Standards and Technology, US Department of Commerce), CODATA Value for the Classical Electron Radius.

[4] Lineweaver, C.H. and Norman, M. (2010) The Potato Radius: A Lower Minimum Size for Dwarf Planets. Proceedings of the 9th Australian Space Science Conference, National Space Society of Australia.

[5] Gott III, J.R., Jurić, M., Schlegel, D., Hoyle, F., Vogeley, M., Tegmark, M., Bahcall, N. and Brinkmann, J. (2005) A Map of the Universe. Astrophysical Journal, 624, 463-484. 